Jean Van Schaftingen · Michel Willem

\title{
Symmetry of solutions of semilinear elliptic problems
}

Received September 15, 2006 and in revised form January 15, 2007

Abstract. We study symmetry properties of least energy positive or nodal solutions of semilinear elliptic problems with Dirichlet or Neumann boundary conditions. The proof is based on symmetrizations in the spirit of Bartsch, Weth and Willem (J. Anal. Math., 2005) together with a strong maximum principle for quasi-continuous functions of Ancona and an intermediate value property for such functions.

Keywords. Polarization, symmetrization, Steiner symmetrization, foliated Schwarz symmetrization, spherical cap symmetrization, quasi-continuous functions, intermediate value theorem, partial symmetry of solutions to semilinear elliptic equations, least energy solutions, ground states, nodal solutions

\section{Introduction}

This work is devoted to the symmetry properties of least energy positive or nodal solutions of the problem

$$
\begin{cases}-\Delta u+a(x) u=g(x, u) & \text { in } \Omega, \\ u=0 & \text { on } \partial \Omega .\end{cases}
$$

The main tools are a general maximum principle and symmetrizations. In Section 2 we prove that if

$$
-\Delta|u|+a|u| \geq 0
$$

in the sense of measures, then either $u=0, u>0$ or $u<0$ almost everywhere. This results from a maximum principle of Ancona [1] (see also Brezis-Ponce [5]), and an intermediate value property for quasi-continuous functions.

In Section 3, we consider the semilinear equation

$$
-\Delta u=f(|x|, u)
$$

J. Van Schaftingen, M. Willem: Département de Mathématique, Université Catholique de Louvain, Chemin du Cyclotron 2, 1348 Louvain-la-Neuve, Belgium;

e-mail: vanschaftingen@math.ucl.ac.be,willem@math.ucl.ac.be

Mathematics Subject Classification (2000): Primary 35J20; Secondary 35A30, 35B50, 35 J60 
on $\Omega$. The main result is Theorem 3.12 which asserts that, under some regularity and growth assumptions on $f$, if $u$ and the polarized functions $u^{H}$ are weak solutions of (1.2) then $u$ has some symmetry properties. The main tools are the maximum principle of Section 2 and approximation of symmetrizations by polarizations.

Section 4 is devoted to the symmetry properties of the least energy positive and nodal solutions of (1.1). In particular we generalize some results of [3, 2, 4, 8, 10, 13]. Our approach is related to [4], but in contrast to [4] we do not assume that the nonlinearity is smooth so that the solutions of (1.1) are merely weak solutions.

\section{Maximum principle}

Consider a function $u \in C(\Omega)$, where $\Omega \subset \mathbb{R}^{N}$ is open and connected. If

$$
-\Delta|u| \geq 0
$$

then by the strong maximum principle, either $|u|=0$ or $|u|>0$. By continuity of $u$, either $u=0, u>0$, or $u<0$. We extend this conclusion to operators of the form $-\Delta+a$, and to the case where $u$ is weakly differentiable and not necessarily continuous.

Proposition 2.1. Let $\Omega \subset \mathbb{R}^{N}, a \in \mathrm{L}_{\mathrm{loc}}^{1}(\Omega)$ and $u \in \mathrm{W}_{\mathrm{loc}}^{1,1}(\Omega)$. If $\Omega$ is open and connected, $a \geq 0, \Delta|u|$ is a Radon measure and

$$
-\Delta|u|+a|u| \geq 0
$$

in the sense that

$$
\int_{E} \Delta|u| \leq \int_{E} a|u| \quad \text { for every Borel set } E \subset \Omega,
$$

then either $u=0, u>0$ or $u<0$ almost everywhere.

Remark 2.2. The assumption $u \in \mathrm{W}_{\text {loc }}^{1,1}(\Omega)$ in the proposition can be weakened to $u \in$ $\mathrm{L}_{\text {loc }}^{1}(\Omega)$ and $u$ being $\mathrm{W}^{1,1}$-quasi-continuous (see Section 2.1 below). Also note that if $\Delta u$ is a Radon measure, then $u$ is $\mathrm{W}^{1,1}$-quasi-continuous and $\Delta|u|$ is a measure [6], so that the conclusion of Proposition 2.1 holds.

Remark 2.3. If $\Delta|u| \in \mathrm{L}_{\text {loc }}^{1}(\Omega)$, then the hypothesis can be stated as

$$
-\Delta|u|+a|u| \geq 0 \quad \text { almost everywhere. }
$$

The proof of Proposition 2.1 relies on the combination of a strong maximum principle, by which either $|u|>0$ outside of a null-capacity set or $|u|=0$, and an intermediate value theorem by which if $|u|>0$ outside of a null-capacity set, then $u$ does not change sign. 


\subsection{Quasi-continuous functions}

The intermediate statements need some basics from capacity theory [11].

Definition 2.4. Let $1 \leq p<\infty, \Omega \subset \mathbb{R}^{N}$ be bounded and open, and $\Sigma \subset \Omega$ be compact. The $\mathrm{W}^{1, p}$-capacity of $\Sigma$ is

$$
\operatorname{cap}_{p, \Omega}(\Sigma)=\inf \left\{\int_{\Omega}|\nabla \varphi|^{p}: \varphi \in \mathcal{D}(\Omega) \text { and } \varphi=1 \text { on a neighbourhood of } \Sigma\right\} .
$$

If $U \subset \Omega$ is open, let

$$
\operatorname{cap}_{p, \Omega}(U)=\sup _{\substack{\Sigma \subset U \\ \Sigma \text { is compact }}} \operatorname{cap}_{p, \Omega}(\Sigma)
$$

For any set $A \subset \Omega$, let

$$
\operatorname{cap}_{p, \Omega}(A)=\inf _{\substack{A \subset U \subset \Omega \\ U \text { is open }}} \operatorname{cap}_{p, \Omega}(U) .
$$

A set $A \subset \Omega$ has null $\mathrm{W}^{1, p}$-capacity if $\operatorname{cap}_{p, \Omega}(A)=0$. A property is said to be true $\mathrm{W}^{1, p}$-quasi-everywhere if the set on which it fails to hold has null $\mathrm{W}^{1, p}$-capacity.

When $q \leq p$, by Hölder's inequality,

$$
\operatorname{cap}_{q, \Omega}(A) \leq \mathcal{L}^{N}(\Omega)^{1-q / p} \operatorname{cap}_{p, \Omega}(A)^{q / p} .
$$

Moreover, the capacity is countably subadditive:

$$
\operatorname{cap}_{p, \Omega}\left(\bigcup_{n \geq 1} A_{n}\right) \leq \sum_{n \geq 1} \operatorname{cap}_{p, \Omega}\left(A_{n}\right) .
$$

While in general the capacity depends on $\Omega$, the notion of vanishing capacity is local and does not depend on $\Omega$.

Proposition 2.5. Let $\Omega \subset \mathbb{R}^{N}$ be bounded and open. If for every $x \in \Omega$, there is an open set $U \subset \Omega$ such that $x \in U$ and $\operatorname{cap}_{p, U}(A \cap U)=0$, then $\operatorname{cap}_{p, \Omega}(A)=0$. Conversely, if $\operatorname{cap}_{p, \Omega}(A)=0$ and $U \subset \Omega$ is open, then $\operatorname{cap}_{p, U}(A \cap U)=0$.

Proof. Since $\Omega \subset \mathbb{R}^{N}$, it has a countable neighbourhood basis; therefore there are open sets $\left(U_{n}\right)_{n \geq 1}$ such that $\Omega=\bigcup_{n \geq 1} U_{n}$ and $\operatorname{cap}_{p, \Omega}\left(A \cap U_{n}\right) \leq \operatorname{cap}_{p, U_{n}}\left(A \cap U_{n}\right)=0$. By countable subadditivity, one concludes that $\operatorname{cap}_{p, \Omega}(A)=0$.

Conversely, by the first part of the proof, we can assume $U=B(x, r) \subset \Omega$. Let $r_{n}=\left(1-n^{-1}\right) r$. By Lemma 2.6 .

$$
\operatorname{cap}_{p, B(x, r)}\left(A \cap B\left(x, r_{n}\right)\right) \leq C_{p} \frac{\operatorname{diam}(\Omega)^{p}}{\left(r-r_{n}\right)^{p}} \operatorname{cap}_{p, \Omega}\left(A \cap B\left(x, r_{n}\right)\right)=0 .
$$

Therefore, by countable subadditivity,

$$
\operatorname{cap}_{p, B(x, r)}(A \cap B(x, r))=0 .
$$


Lemma 2.6. Let $0<\rho<r$. If $A \subset B(x, \rho)$ and $B(x, r) \subset \Omega$, then

$$
\operatorname{cap}_{p, B(x, r)}(A) \leq C_{p} \frac{\operatorname{diam}(\Omega)^{p}}{(r-\rho)^{p}} \operatorname{cap}_{p, \Omega}(A) .
$$

Proof. It is sufficient to prove the inequality when $A$ is compact. For $\varepsilon>0$, let $\varphi \in \mathcal{D}(\Omega)$ be such that $\varphi \leq 1$ in $\Omega, \varphi \equiv 1$ on a neighbourhood of $A$ and

$$
\operatorname{cap}_{p, \Omega}(A) \geq \int_{\Omega}|\nabla \varphi|^{p}-\varepsilon
$$

Consider $\eta \in \mathcal{D}(B(x, r))$ such that $\eta \leq 1$ in $B(x, r), \eta \equiv 1$ on $B(x, \rho)$ and $\|\nabla \eta\|_{\mathrm{L}^{\infty}} \leq$ $2 /(r-\rho)$. Then $\eta \varphi \in \mathcal{D}(B(x, r))$ and $\eta \varphi \equiv 1$ on a neighbourhood of $A$, so that

$$
\begin{aligned}
\operatorname{cap}_{p, B(x, r)}(A) & \leq \int_{B(x, r)}|\nabla(\eta \varphi)|^{p} \leq 2^{p-1}\left(\int_{B(x, r)}|\nabla \eta|^{p}|\varphi|^{p}+\int_{B(x, r)}|\eta|^{p}|\nabla \varphi|^{p}\right) \\
& \leq 2^{p-1}\left(\frac{2^{p}}{(r-\rho)^{p}} \int_{\Omega}|\varphi|^{p}+\int_{\Omega}|\nabla \varphi|^{p}\right) .
\end{aligned}
$$

By the Poincaré inequality, this implies

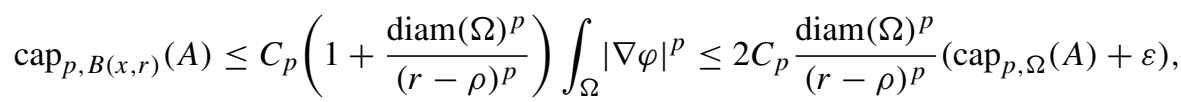

because of $|r-\rho| \leq r \leq \operatorname{diam}(\Omega) / 2$. Since $\varepsilon>0$ is arbitrary, this concludes the proof.

Definition 2.7. If $\Omega \subset \mathbb{R}^{N}$ is bounded, a function $u: \Omega \rightarrow \mathbb{R}$ is $\mathrm{W}^{1, p}$-quasi-continuous if there is a sequence $\left(\omega_{n}\right)_{n \geq 1}$ of $\Omega$ of open subsets such that $\left.u\right|_{\Omega \backslash \omega_{n}}$ is continuous and $\operatorname{cap}_{p, \Omega}\left(\omega_{n}\right) \rightarrow 0$ as $n \rightarrow \infty$.

Example 2.8. Every function $u \in \mathrm{W}_{\text {loc }}^{1, p}(\Omega)$ is $\mathrm{W}^{1, p}$-quasi-continuous 11$]$.

Just as null-capacity, quasi-continuity is a local notion:

Proposition 2.9. Let $\Omega \subset \mathbb{R}^{N}$ be bounded and open and let $u: \Omega \rightarrow \mathbb{R}$. If for every $x \in \Omega$, there is an open set $U \subset \Omega$ such that $x \in U$ and $\left.u\right|_{U}$ is quasi-continuous, then $u$ is quasi-continuous. Conversely, if $u$ is quasi-continuous and $U \subset \Omega$ is open, then $\left.u\right|_{U}$ is quasi-continuous.

Proof. Since $\Omega \subset \mathbb{R}^{N}$, it has a countable neighbourhood basis, so there are open sets $\left(U_{n}\right)_{n \geq 1}$ such that $\Omega=\bigcup_{n \geq 1} U_{n}$ and $\left.u\right|_{U_{n}}$ is quasi-continuous. Let $\varepsilon>0$. By definition of

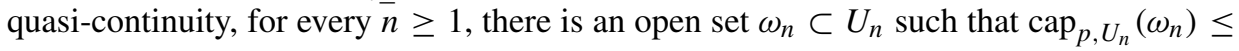
$2^{-n} \varepsilon$ and $\left.u\right|_{U_{n} \backslash \omega_{n}}$ is continuous. Let $\omega=\bigcup_{n>1} \omega_{n}$. The set $\omega$ is open. The function $\left.u\right|_{\Omega \backslash \omega}$ is continuous. By countable subadditivity of the capacity, $\operatorname{cap}_{p, \Omega}(\omega) \leq \varepsilon$. Since $\varepsilon>0$ is arbitrary, $u$ is quasi-continuous. 
Conversely, by the first part of the proof, we can assume $U=B(x, r) \subset \Omega$. Let $\varepsilon>0$. By definition of quasi-continuity, there is $\omega_{n}$ such that $\left.u\right|_{\Omega \backslash \omega_{n}}$ is continuous and $\operatorname{cap}_{p, \Omega}\left(\omega_{n}\right) \leq \varepsilon 3^{-n}$. Letting $r_{n}=\left(1-2^{-n / p}\right) r$, one has

$$
\begin{aligned}
& \operatorname{cap}_{p, B(x, r)}\left(\omega_{n} \cap B\left(x, r_{n}\right)\right) \\
& \quad \leq C_{p} \frac{\operatorname{diam}(\Omega)^{p}}{\left(r-r_{n}\right)^{p}} \operatorname{cap}_{p, \Omega}\left(\omega_{n} \cap B\left(x, r_{n}\right)\right) \leq C_{p} \varepsilon \frac{\operatorname{diam}(\Omega)^{p}}{r^{p}} \frac{2^{n}}{3^{n}} .
\end{aligned}
$$

Let now $\omega=\bigcup_{n \geq 1}\left(\omega_{n} \cap B\left(x, r_{n}\right)\right)$. The function $\left.u\right|_{B(x, r) \backslash \omega}$ is continuous, and $\operatorname{cap}_{p, B(x, r)}(\omega) \leq C_{p} 2 \varepsilon \operatorname{diam}(\Omega)^{p} / r^{p}$. Since $\varepsilon>0$ was arbitrary, $\left.u\right|_{B(x, r)}$ is quasicontinuous.

\subsection{Maximum principle}

The first ingredient of the proof of Proposition 2.1 is the following strong maximum principle.

Theorem 2.10 (Ancona [1], Brezis and Ponce [5]). Let $\Omega$ be bounded and connected. Let $u \in \mathrm{L}^{1}(\Omega)$ with $u \geq 0$ almost everywhere in $\Omega$ be such that $\Delta u$ is a Radon measure on $\Omega$. Then there exists $\tilde{u} \mathrm{~W}^{1,2}$-quasi-continuous such that $u=\tilde{u}$ almost everywhere in $\Omega$.

Let $a \in \mathrm{L}^{1}(\Omega)$ with $a \geq 0$ almost everywhere in $\Omega$. If

$$
-\Delta u+a u \geq 0 \quad \text { in } \Omega
$$

in the sense that

$$
\int_{E} \Delta u \leq \int_{E} a u \quad \text { for every Borel set } E \subset \Omega,
$$

and if $\tilde{u}=0$ on a set of positive $\mathrm{W}^{1,2}$-capacity in $\Omega$, then $u=0$ almost everywhere in $\Omega$.

\subsection{Intermediate value property}

The second ingredient is an intermediate value property for $\mathrm{W}^{1,1}$-quasi-continuous functions.

Proposition 2.11. Let $\Omega$ be open, connected and bounded. If $u: \Omega \rightarrow \mathbb{R}$ is $\mathrm{W}^{1,1}$-quasicontinuous and $u \neq 0 \mathrm{~W}^{1,1}$-quasi-everywhere, then either $u>0$ or $u<0$ almost everywhere.

Remark 2.12. Recall that null sets for the $\mathrm{W}^{1,1}$-capacity and for the $N$ - 1-dimensional Hausdorff measure coincide [11]. Therefore, Proposition 2.11 can be restated as: If $u \neq 0$ almost everywhere for the $N-1$-dimensional Hausdorff measure, then $u$ does not change sign. When $u$ is continuous, this can be proved straightforwardly. 
Remark 2.13. In the same spirit, if $\Omega$ is connected, $u \in \mathrm{W}_{\text {loc }}^{1,1}(\Omega)$ and $|u| \geq \varepsilon>0$ almost everywhere, then either $u \geq \varepsilon$ or $u \leq-\varepsilon$ almost everywhere [9].

The proof of the intermediate value property of Proposition 2.11 relies on the following lemma, which is a geometric version of the Poincaré inequality.

Lemma 2.14. Let $N \geq 1$. There is $C>0$ such that if $F^{+}, F^{-} \subset B(0,1) \subset \mathbb{R}^{N}$ are disjoint and closed, then

$$
\min \left(\mathcal{L}^{N}\left(F^{+}\right), \mathcal{L}^{N}\left(F^{-}\right)\right)^{(N-1) / N} \leq C \operatorname{cap}_{1, B(0,1)}\left(B(0,1) \backslash\left(F^{+} \cup F^{-}\right)\right) .
$$

Proof. Let $S=B(0,1) \backslash\left(F^{+} \cup F^{-}\right)$and choose $(2 / 3)^{1 / N} \leq \rho \leq 1$ such that

$$
\mathcal{L}^{N}\left(F^{+} \cap B(0, \rho)\right) \geq \mathcal{L}^{N}\left(F^{+}\right) / 2, \quad \mathcal{L}^{N}\left(F^{-} \cap B(0, \rho)\right) \geq \mathcal{L}^{N}\left(F^{-}\right) / 2 .
$$

Since the sets $F^{+} \cap B[0, \rho]$ and $F^{-} \cap B[0, \rho]$ are compact, there are open disjoint subsets $U^{+}$and $U^{-}$of $B(0,1)$ such that

$$
F^{+} \cap B[0, \rho] \subset U^{+}, \quad F^{-} \cap B[0, \rho] \subset U^{-} .
$$

Without loss of generality, assume that $\mathcal{L}^{N}\left(U^{+}\right) \leq \mathcal{L}^{N}(B(0,1)) / 2$. Let $\Sigma=B[0, \rho] \backslash$ $\left(U_{+} \cup U_{-}\right)$and note that $\Sigma \subset S$.

Since $\Sigma \subset B(0,1)$ is compact, there exists $\varphi \in \mathcal{D}(B(0,1))$ such that $\varphi \leq 1, \varphi=1$ on a neighbourhood of $\Sigma$ and

$$
\int_{B(0,1)}|\nabla \varphi| \leq 2 \operatorname{cap}_{1, B(0,1)}(\Sigma) \leq 2 \operatorname{cap}_{1, B(0,1)}(S) .
$$

Define $\psi: B[0, \rho] \rightarrow \mathbb{R}$ by

$$
\psi(x)= \begin{cases}\varphi(x) & \text { if } x \in U^{-} \cup \Sigma \\ 1 & \text { if } x \in U^{+}\end{cases}
$$

for $x \in B[0, \rho]$. By the Poincaré-Sobolev inequality

$$
\left(\int_{B(0, \rho)}\left|\psi-f_{B(0, \rho)} \psi\right|^{N /(N-1)}\right)^{(N-1) / N} \leq d_{N} \rho \int_{B(0, \rho)}|\nabla \psi| \leq d_{N} \operatorname{cap}_{1, B(0,1)}(S),
$$

where $d_{N}$ depends only on $N$. Moreover,

$$
\begin{aligned}
\int_{B(0, \rho)} \psi & =\mathcal{L}^{N}\left(B(0, \rho) \cap U^{+}\right)+\int_{B(0, \rho) \backslash U^{+}} \varphi \leq \mathcal{L}^{N}\left(U^{+}\right)+\int_{B(0,1)} \varphi \\
& \leq(3 / 4) \mathcal{L}^{N}(B(0, \rho))+c_{N} \operatorname{cap}_{1, B(0,1)}(S)
\end{aligned}
$$

where $c_{N}$ comes from the Poincaré inequality in $\mathrm{W}_{0}^{1,1}(B(0,1))$. 
Let now $\eta=1 /\left(20 c_{N}\right)$. If $\operatorname{cap}_{1, B(0,1)}(S) \geq \eta$, then one has the trivial bound

$$
\mathcal{L}^{N}\left(F^{+}\right) \leq \mathcal{L}^{N}(B(0,1)) \leq \mathcal{L}^{N}(B(0,1))\left(\frac{\operatorname{cap}_{1, B(0,1)}(S)}{20 c_{N}}\right)^{N /(N-1)},
$$

while if $\operatorname{cap}_{1, B(0,1)}(S) \leq \eta$, then

$$
f_{B(0, \rho)} \psi \leq 3 / 4+1 / 20=4 / 5
$$

and

$$
\begin{aligned}
\frac{\mathcal{L}^{N}\left(F^{+}\right)}{2} \leq \mathcal{L}^{N}\left(U^{+}\right) & \leq 5^{N /(N-1)} \int_{B(0, \rho)}\left|\psi-f_{B(0, \rho)} \psi\right|^{N /(N-1)} \\
& \leq\left(5 d_{N} \operatorname{cap}_{1, B(0,1)}(S)\right)^{N /(N-1)}
\end{aligned}
$$

We can now prove Proposition 2.11 .

Proof of Proposition 2.11. Assume first that $\Omega=B(0,1)$. Since $u$ is quasi-continuous and is nonzero quasi-everywhere, by definition, for every $\varepsilon>0$, there exists an open set $\omega \subset B(0,1)$ such that $\left.u\right|_{B(0,1) \backslash \omega}$ is continuous and does not vanish, and cap ${ }_{1, B(0,1)}(\omega)$ $\leq \varepsilon$. Define

$$
B^{+}=\{x \in B(0,1): u(x) \geq 0\}, \quad B^{-}=\{x \in B(0,1): u(x) \leq 0\},
$$

and let $F^{+}=B^{+} \backslash \omega$ and $F^{-}=B^{-} \backslash \omega$. Since the sets $F^{+}$and $F^{-}$are closed and disjoint in $B(0,1)$, by Lemma 2.6 , they satisfy

$$
\min \left(\mathcal{L}^{N}\left(F^{+}\right), \mathcal{L}^{N}\left(F^{-}\right)\right) \leq C \varepsilon^{N /(N-1)} .
$$

On the other hand, by the Poincaré-Sobolev inequality,

$$
\mathcal{L}^{N}(\omega) \leq C^{\prime} \varepsilon^{N /(N-1)} .
$$

Therefore,

$$
\min \left(\mathcal{L}^{N}\left(B^{+}\right), \mathcal{L}^{N}\left(B^{-}\right)\right) \leq \min \left(\mathcal{L}^{N}\left(F^{+}\right), \mathcal{L}^{N}\left(F^{-}\right)\right)+\mathcal{L}^{N}(\omega) \leq\left(C+C^{\prime}\right) \varepsilon^{N /(N-1)} .
$$

Since $\varepsilon>0$ is arbitrary, either $\mathcal{L}^{N}\left(B^{+}\right)=0$ or $\mathcal{L}^{N}\left(B^{-}\right)=0$. This extends to $\Omega=$ $B(x, r)$ by translation and dilation.

In general let

$$
\begin{aligned}
& \Omega^{+}=\{x \in \Omega: \exists r>0 \text { such that } u(y)>0 \text { for a.e. } y \in B(x, r)\}, \\
& \Omega^{-}=\{x \in \Omega: \exists r>0 \text { such that } u(y)<0 \text { for a.e. } y \in B(x, r)\} .
\end{aligned}
$$

The sets $\Omega^{-}$and $\Omega^{+}$are open and disjoint by definition. By the first part of the proof, $\Omega=\Omega^{+} \cup \Omega^{-}$. Since $\Omega$ is connected, either $\Omega=\Omega^{+}$or $\Omega=\Omega^{-}$. 


\subsection{Proof of Proposition 2.1}

First assume that $\Omega$ is bounded and $u \in \mathrm{L}^{1}(\Omega)$. By Theorem 2.10 either $|u|=0$ almost everywhere or $|u|>0, \mathrm{~W}^{1,2}$-quasi-everywhere. If $|u|>0, \mathrm{~W}^{1,2}$-quasi-everywhere, then by $2.2, u \neq 0 \mathrm{~W}^{1,1}$-quasi-everywhere, and then by Proposition 2.11 either $u>0$ or $u<0$ almost everywhere.

In general, define

$$
\begin{aligned}
& \Omega^{+}=\{x \in \Omega: \exists \overline{B(x, r)} \subset \Omega \text { such that } u(y)>0 \text { for a.e. } y \in B(x, r)\}, \\
& \Omega^{0}=\{x \in \Omega: \exists \overline{B(x, r)} \subset \Omega \text { such that } u(y)=0 \text { for a.e. } y \in B(x, r)\}, \\
& \Omega^{-}=\{x \in \Omega: \exists \overline{B(x, r)} \subset \Omega \text { such that } u(y)<0 \text { for a.e. } y \in B(x, r)\} .
\end{aligned}
$$

By definition $\Omega^{+}, \Omega^{0}$ and $\Omega^{-}$are open and disjoint. Moreover, since the first part of the proof is applicable to $B(x, r)$ when $\overline{B(x, r)} \subset \Omega$, one has

$$
\Omega=\Omega^{+} \cup \Omega^{0} \cup \Omega^{-} \text {. }
$$

By the connectedness of $\Omega$, one of these sets must be $\Omega$.

\section{Invariance under symmetrizations}

\subsection{Symmetrizations and polarizations}

Our symmetry results are expressed in terms of symmetrizations and proved in terms of polarizations.

3.1.1. Symmetrization. Let us first recall the notion of Steiner and cap symmetrizations of sets and of functions.

Definition 3.1. Let $S$ be a $k$-dimensional affine subspace of $\mathbb{R}^{N}, 0 \leq k \leq N-1$. The Steiner symmetrization of a set $A \subset \mathbb{R}^{N}$ with respect to $S$ is the unique set $A^{*}$ such that for any $x \in S$, if $L$ is the $(N-k)$-dimensional hyperplane orthogonal to $S$ that contains $x$, then

$$
A^{*} \cap L=B(x, r) \cap L,
$$

where $0 \leq r \leq \infty$ is defined by $\mathcal{H}^{N-k}(B(x, r) \cap L)=\mathcal{H}^{N-k}(A \cap L)$.

Definition 3.2. Let $S$ be a $k$-dimensional closed affine half subspace of $\mathbb{R}^{N}, 1 \leq k \leq$ $N-1$ and let $\partial S$ be the boundary of $S$ inside the affine plane generated by $S$. The cap symmetrization of a set $A \subset \mathbb{R}^{N}$ with respect to $S$ is the unique set $A^{*}$ such that $A^{*} \cap \partial S=$ $A \cap \partial S$ and for each $x \in \partial S$, if $L$ is the $(N-k+1)$-dimensional hyperplane orthogonal to $\partial S$ that contains $x$ and $y$ is the unique point of the intersection $\partial B(x, \varrho) \cap S \cap L$, then, for every $\varrho>0$,

$$
A^{*} \cap \partial B(x, \varrho) \cap L=B(y, r) \cap \partial B(x, \varrho) \cap L,
$$

where $r \geq 0$ is defined by $\mathcal{H}^{N-k}(B(y, r) \cap \partial B(x, \varrho) \cap L)=\mathcal{H}^{N-k}(A \cap \partial B(x, \varrho) \cap L)$. 
From now on, $S$ is a fixed affine closed subspace or half subspace so that $*$ denotes a fixed Steiner or cap symmetrization. The function $u: \Omega \rightarrow \overline{\mathbb{R}}$ is admissible with respect to $*$ if

(i) $\Omega^{*}=\Omega$,

(ii) for every $c>0$,

$$
\mathcal{L}^{N}(\{x \in \Omega:|u(x)|>c\})<\infty
$$

(iii) one of the following conditions is satisfied:

(a) $u \geq 0$,

(b) $S$ is a half subspace and $\left(\mathbb{R}^{N} \backslash \Omega\right)^{*}=\mathbb{R}^{N} \backslash \Omega$.

Definition 3.3. Let $\Omega \subset \mathbb{R}^{N}$ and $u: \Omega \rightarrow \overline{\mathbb{R}}$ be admissible with respect to $*$. The symmetrization of $u$ is the unique function $u^{*}$ such that for each $c \in \overline{\mathbb{R}}$,

$$
\left\{x \in \Omega: u^{*}(x)>c\right\}=\{x \in \Omega: u(x)>c\}^{*} .
$$

The symmetrization has many interesting properties that we shall not use in this article [7, 12, 18, 19].

3.1.2. Polarization. For every closed affine halfspace $H$, let $\sigma_{H}$ denote the reflection with respect to the boundary $\partial H$.

Definition 3.4. Let $H \subset \mathbb{R}^{N}$ be a closed affine halfspace, $\Omega \subset \mathbb{R}^{N}$ such that $\sigma_{H}(\Omega)=\Omega$ and $u: \Omega \rightarrow \overline{\mathbb{R}}$. The polarization of $u$ with respect to $H$ is the function $u^{H}: \mathbb{R}^{N} \rightarrow \mathbb{R}$ defined by

$$
u^{H}(x)= \begin{cases}\max \left(u(x), u\left(\sigma_{H}(x)\right)\right) & \text { if } x \in H, \\ \min \left(u(x), u\left(\sigma_{H}(x)\right)\right) & \text { if } x \notin H .\end{cases}
$$

The polarization $A^{H}$ of a set $A \subset \mathbb{R}^{N}$ is defined by

$$
\chi_{A^{H}}=\left(\chi_{A}\right)^{H} \text {, }
$$

where $\chi_{A}$ is the characteristic function of $A$, whose value is 1 in A and 0 elsewhere.

If $j$ is an isometry such that $j(\Omega)=\sigma_{H}(\Omega)=\Omega$ and $u: \Omega \rightarrow \mathbb{R}$, then

$$
(u \circ j)^{H}=u^{j^{-1}(H)} \circ j \text {. }
$$

Remark 3.5. The polarization can also be defined as the cap symmetrization with respect to $H$ [16].

If $*$ is the symmetrization with respect to $S$, the set of polarizations that generates the symmetrization $*$ is

$$
\mathcal{H}_{*}=\left\{H \subset \mathbb{R}^{N}: H \text { is a closed affine halfspace, } \partial S \subset \partial H \text { and } S \subset H\right\} .
$$

First note that if $u$ is admissible for $*$, then

$$
u^{*}=u \quad \text { if and only if } \quad \forall H \in \mathcal{H}_{*}, u^{H}=u .
$$

In fact the polarizations of $\mathcal{H}_{*}$ can be used to approximate $*$ : 
Theorem 3.6. There exists $\left(H_{n}\right)_{n \geq 1}$ such that for every $1 \leq p<\infty$ and for every $u \in \mathrm{L}^{p}(\Omega)$, if $u$ is admissible for $*$, then

$$
u^{H_{1} \ldots H_{n}} \rightarrow u^{*} \quad \text { in } \mathrm{L}^{p}(\Omega) \text { as } n \rightarrow \infty
$$

Similarly, if $u \in C(\Omega)$ is admissible for $*$ and $\lim _{|x| \rightarrow \infty} u(x)=0$,

$$
u^{H_{1} \ldots H_{n}} \rightarrow u^{*} \quad \text { uniformly as } n \rightarrow \infty
$$

Remark 3.7. Theorem 3.6 is proved in [17]. Slightly weaker results were obtained before [7, 15, 19]. In fact random sequences of polarizations converge almost surely [16].

Definition 3.8. A set $\Omega \subset \mathbb{R}^{N}$ is totally invariant (with respect to $*$ ) if $\Omega^{*}=\Omega$ and $\left(\mathbb{R}^{N} \backslash \Omega\right)^{*}=\mathbb{R}^{N} \backslash \Omega$, or equivalently, if $\sigma_{H}(\Omega)=\Omega$ for every $H \in \mathcal{H}_{*}$.

We can extend the notion of $u^{*}=u$ to nonadmissible functions by relying on the equivalence 3.1 :

Definition 3.9. If $\Omega$ is totally invariant, a function $u: \Omega \rightarrow \mathbb{R}$ is invariant under the symmetrization $*$ if for every $H \in \mathcal{H}_{*}$,

$$
u^{H}=u \text {. }
$$

If $-u$ is also invariant, then $u$ is totally invariant with respect to $*$.

3.1.3. Isometries. Finally, we consider the group of isometries which are compatible with a symmetrization.

Definition 3.10. Let $*$ be the symmetrization with respect to $S$. The group $G_{*}$ of isometries compatible with $*$ is

$$
G_{*}=\left\{i: \mathbb{R}^{N} \rightarrow \mathbb{R}^{N}: i \text { is an isometry and } \forall A \subset \mathbb{R}^{N}, i(A)^{*}=A^{*}\right\}
$$

When $*$ is the Steiner symmetrization with respect to $H, G_{*}$ is generated by the isometries that leave invariant all the points $S$ and by translations perpendicular to $S$, while if $*$ is the cap symmetrization with respect to $S$, then $G_{*}$ is the group of isometries for which all the points of $\partial S$ are invariant. In both cases $G_{*}$ is generated by $\left\{\sigma_{H}: H \in \mathcal{H}_{*}\right\}$. Therefore:

Proposition 3.11. The set $\Omega \subset \mathbb{R}^{N}$ is totally invariant if and only if $i(\Omega)=\Omega$ for every $i \in G_{*}$. 


\subsection{Invariance under symmetrization}

We can now state the main symmetry result.

Theorem 3.12. Let $*$ be the symmetrization with respect to $S, \Omega \subset \mathbb{R}^{N}$ be open, connected and totally invariant with respect to $*$, and $f: \Omega \times \mathbb{R} \rightarrow \mathbb{R}$ be a Carathéodory function. Assume that

(i) there exists $g \in \mathrm{L}_{\text {loc }}^{1}(\Omega)$ with $g \leq 0$ and a measurable locally bounded function $c: \Omega \rightarrow \mathbb{R}^{-}$such that for almost every $x \in \Omega$, for $s \geq t$,

$$
f(x, s)-f(x, t) \geq\left(g(x)+c(x)\left(|s|^{p}+|t|^{p}\right)\right)(s-t),
$$

(ii) for every $t \in \mathbb{R}, f(\cdot, t)$ is invariant under the symmetrization $*$.

Let $u \in \mathrm{L}_{\mathrm{loc}}^{p}(\Omega)$ be admissible for $*$, such that $f(x, u) \in \mathrm{L}_{\mathrm{loc}}^{1}(\Omega)$, and, in the sense of distributions,

$$
-\Delta u=f(x, u) .
$$

If for every $H \in \mathcal{H}_{*}, f\left(x, u^{H}\right) \in \mathrm{L}_{\text {loc }}^{1}(\Omega)$ and

$$
-\Delta u^{H}=f\left(x, u^{H}\right)
$$

then there exists $i \in G_{*}$ such that $u^{*}=u \circ i$.

Remark 3.13. If for all $i \in G_{*}$, all $t \in \mathbb{R}$ and almost every $x \in \Omega$,

$$
f(i(x), t)=f(x, t),
$$

then the hypothesis (ii) holds.

Proof of Theorem 3.12 By Proposition 3.14 below, for every $H \in \mathcal{H}_{*}$, there is an isometry $j \in G_{*}$ such that $u^{H}=u \circ j$. Hence, by Proposition 3.15, there exists $i \in G_{*}$ such that $u^{*}=u \circ i$.

\subsection{Invariance under polarizations}

The first step in the proof of Theorem 3.12 is to establish a variant of Theorem 3.12 for polarizations.

Proposition 3.14. Let $H \subset \mathbb{R}^{N}$ be a halfspace, and $\Omega \subset \mathbb{R}^{N}$ be open, connected and such that $\sigma_{H}(\Omega)=\Omega$. Let $f: \Omega \times \mathbb{R} \rightarrow \mathbb{R}$ be a Carathéodory function. Assume

(i) there is $g \in \mathrm{L}_{\text {loc }}^{1}(\Omega), g \leq 0$ and a measurable locally bounded function $c: \Omega \rightarrow \mathbb{R}^{-}$ such that for almost every $x \in \Omega$, for $s \geq t$,

$$
f(x, s)-f(x, t) \geq\left(g(x)+c(x)\left(|s|^{p}+|t|^{p}\right)\right)(s-t),
$$


(ii) for almost every $x \in \Omega \cap H$, for every $t$,

$$
f\left(\sigma_{H}(x), t\right) \leq f(x, t) .
$$

Let $u \in \mathrm{L}_{\mathrm{loc}}^{p}(\Omega)$ be such that $f(x, u) \in \mathrm{L}_{\mathrm{loc}}^{1}(\Omega)$ and

$$
-\Delta u=f(x, u) .
$$

If $f\left(x, u^{H}\right) \in \mathrm{L}_{\mathrm{loc}}^{1}(\Omega)$ and

$$
-\Delta u^{H}=f\left(x, u^{H}\right)
$$

(in the sense of distributions), then either $u^{H}=u$ or $u^{H}=u \circ \sigma_{H}$.

Proof. First note that for every $x \in \Omega \cap H$,

$$
\left|u-u \circ \sigma_{H}\right|(x)=2 u^{H}(x)-u(x)-u \circ \sigma_{H}(x) .
$$

By the equations that $u$ and $u^{H}$ satisfy and (ii), one has

$$
\begin{aligned}
-\Delta\left|u-u \circ \sigma_{H}\right|(x) & =f\left(x, u^{H}(x)\right)-f(x, u(x))+f\left(x, u^{H}(x)\right)-f\left(\sigma_{H}(x), u\left(\sigma_{H}(x)\right)\right) \\
& \geq f\left(x, u^{H}(x)\right)-f(x, u(x))+f\left(x, u^{H}(x)\right)-f\left(x, u\left(\sigma_{H}(x)\right)\right) .
\end{aligned}
$$

Using (i), and remembering that either $u^{H}(x)=u(x)$ or $u^{H}(x)=u\left(\sigma_{H}(x)\right)$, we have

$$
\begin{aligned}
-\Delta\left|u-u \circ \sigma_{H}\right|(x) \geq & \left(g(x)+c(x)\left(\left|u^{H}(x)\right|^{p}+|u(x)|^{p}\right)\right)\left(u^{H}(x)-u(x)\right) \\
& +\left(g(x)+c(x)\left(\left|u^{H}(x)\right|^{p}+\left|u \circ \sigma_{H}(x)\right|^{p}\right)\right)\left(u^{H}(x)-u \circ \sigma_{H}(x)\right) \\
= & \left(g(x)+c(x)\left(|u(x)|^{p}+\left|u \circ \sigma_{H}(x)\right|^{p}\right)\right)\left|u(x)-u \circ \sigma_{H}(x)\right| .
\end{aligned}
$$

Since

$$
g+c\left(|u|^{p}+\left|u \circ \sigma_{H}\right|^{p}\right) \in \mathrm{L}_{\mathrm{loc}}^{1}\left(\mathbb{R}^{N}\right),
$$

since $\Delta u$ and $\Delta u^{H}$ are locally integrable and since $\Omega \cap H$ is connected, Proposition 2.1 is applicable, and so either $u-u \circ \sigma_{H}>0, u-u \circ \sigma_{H}=0$ or $u-u \circ \sigma_{H}<0$ in $\Omega \cap H$. The conclusion follows.

\subsection{From polarizations to symmetrizations}

We now show how the conclusion of Theorem 3.12 can be deduced from that of Proposition 3.14 .

Proposition 3.15. Let $\Omega \subset \mathbb{R}^{N}$ be such that $\Omega^{*}=\Omega$ and let $u: \Omega \rightarrow \mathbb{R}$ be admissible for $*$. If for every $H \in \mathcal{H}_{*}$, there exists $j \in G_{*}$ such that $u^{H}=u \circ j$ then there exists $i \in G_{*}$ such that $u^{*}=u \circ i$. 
Proof. The assertion is trivial when $u=0$, so assume $u \neq 0$. Let $\left(H_{n}\right)_{n \geq 1}$ be the sequence of polarizations given by Theorem 3.6 We first claim that for every $n \geq 0$, there is $i_{n} \in G_{*}$ such that

$$
u^{H_{1} \ldots H_{n}}=u \circ i_{n} .
$$

This is clearly true with $i_{0}=\mathrm{id}$ when $n=0$. If it is true for $n \geq 0$, then

$$
u^{H_{1} \ldots H_{n+1}}=\left(u \circ i_{n}\right)^{H_{n+1}}=u^{i_{n}^{-1}\left(H_{n+1}\right)} \circ i_{n}=u \circ j_{n+1} \circ i_{n},
$$

with $j_{n+1}$ given by the hypothesis, so that the assertion is proved with $i_{n+1}=j_{n+1} \circ i_{n}$ $\in G_{*}$.

For $\lambda>1$ and $t \in \mathbb{R}$, define

$$
h_{\lambda}(t)= \begin{cases}-\lambda+1 / \lambda & \text { if } t \leq-\lambda, \\ t+1 / \lambda & \text { if }-\lambda \leq t \leq-1 / \lambda, \\ 0 & \text { if }-1 / \lambda \leq t \leq 1 / \lambda, \\ t-1 / \lambda & \text { if } 1 / \lambda \leq t \leq \lambda, \\ \lambda-1 / \lambda & \text { if } t \geq \lambda\end{cases}
$$

For $x \in \Omega$, define $u_{\lambda}=h_{\lambda} \circ u$, and note that $u_{\lambda} \in \mathrm{L}^{1}(\Omega),\left(u_{\lambda}\right)^{*}=h_{\lambda} \circ u^{*}$ and

$$
\left(u_{\lambda}\right)^{H_{1} \ldots H_{n}}=u_{\lambda} \circ i_{n} .
$$

We now claim that for every bounded set $B \subset \Omega$, the sequence $\left(\left.i_{n}\right|_{B}\right)_{n \geq 1}$ is bounded. If $u^{+} \neq 0$, then there is $\lambda$ such that $v=u_{\lambda}^{+} \neq 0$. Without loss of generality, assume $B=B^{*}$ and

$$
\int_{B} v>\frac{1}{2} \int_{\Omega} v .
$$

By elementary properties of symmetrizations (the Hardy-Littlewood inequality and the Cavalieri principle [7, 12, 18, 19]),

$$
\int_{B} v^{*} \geq \int_{B} v>\frac{1}{2} \int_{\Omega} v=\frac{1}{2} \int_{\Omega} v^{*}
$$

If $i_{n}(B) \cap B \neq \emptyset$, then $B \cap i_{n}^{-1}(B) \neq \emptyset$ and

$$
\begin{aligned}
\int_{\Omega}\left|v^{*}-v \circ i_{n}\right| & \geq \int_{B \cup i_{n}^{-1}(B)}\left|v^{*}-v \circ i_{n}\right| \geq \int_{B} v^{*}-\int_{i_{n}(B)} v+\int_{B} v-\int_{i_{n}^{-1}(B)} v^{*} \\
& \geq \int_{B} v^{*}-\int_{\Omega \backslash B} v+\int_{B} v-\int_{\Omega \backslash B} v^{*}>0 .
\end{aligned}
$$

In view of the convergence $v \circ i_{n} \rightarrow v^{*}$ in $\mathrm{L}^{1}(\Omega)$, this proves that there exists $n_{0}$ such that $i_{n}^{-1}(B) \cap B \neq \emptyset$ for $n>n_{0}$. The claim is thus proven when $u^{+} \neq 0$. In the case where $u^{+}=0$, a similar reasoning for $w=u_{\lambda}^{-}$yields the same conclusion.

Since $i_{n}$ is a bounded sequence of isometries, it is relatively compact. Choose $i \in G_{*}$ and $\left(i_{n_{k}}\right)_{k \geq 1}$ such that $i_{n_{k}} \rightarrow i$. Therefore, for every $\lambda>1, u_{\lambda} \circ i_{n_{k}} \rightarrow u_{\lambda} \circ i$ in $\mathrm{L}^{1}(\Omega)$. Since $u_{\lambda} \circ i_{n} \rightarrow u_{\lambda}^{*}$ in $\mathrm{L}^{1}(\Omega)$, one has $u_{\lambda}^{*}=u_{\lambda} \circ i$. Letting $\lambda \rightarrow \infty$, one concludes that $u^{*}=u \circ i$. 


\section{Symmetry of solutions}

\subsection{Symmetry of minimizers}

Let $*$ be the symmetrization associated to $S$ and let $\Omega \subset \mathbb{R}^{N}$ be an open subset such that $\Omega^{*}=\Omega$ and $\left(\mathbb{R}^{N} \backslash \Omega\right)^{*}=\mathbb{R}^{N} \backslash \Omega$.

We consider the problem

$$
\begin{cases}-\Delta u+a(x) u=g(x, u) & \text { in } \Omega \\ u=0 & \text { on } \partial \Omega\end{cases}
$$

We assume that

$\left(A_{1}\right) a \in \mathrm{L}_{\text {loc }}^{1}(\Omega), g=\Omega \times \mathbb{R} \rightarrow \mathbb{R}$ is a Carathéodory function,

$\left(A_{2}\right) g(x, t)=o(t)$ as $t \rightarrow 0$, uniformly in $x \in \Omega$,

$\left(A_{3}\right)$ there exists $2<p \leq 2^{*}$ (if $N \geq 3$ ) or $2<p<\infty$ (if $N=2$ ) and $C \geq 0$ such that, for any $t \in \mathbb{R}$ and $x \in \Omega$,

$$
|g(x, t)| \leq C\left(1+|t|^{p-1}\right)
$$

$\left(A_{4}\right)$ for almost every $x \in \Omega$, the function $t \mapsto g(x, t) /|t|$ is nondecreasing for $t \neq 0$ and (strictly) increasing for $|t|>0$ small,

$\left(A_{5}\right)$ for every $t \in \mathbb{R}, g(\cdot, t)$ is invariant under the symmetrization $*$,

$\left(A_{6}\right)$ there exists $c>0$ such that for every $u \in \mathcal{D}(\Omega)$,

$$
\int_{\Omega}\left(|\nabla u|^{2}+a u^{2}\right) \geq c \int_{\Omega}|\nabla u|^{2} .
$$

Let

$$
\|u\|^{2}=\int_{\Omega}\left(|\nabla u|^{2}+a u^{2}\right)
$$

and $X$ be the completion of $\mathcal{D}(\Omega)$ with respect to the norm $\|\cdot\|$. Let us define on $X$,

$$
\Phi(u)=\int_{\Omega}\left(\frac{|\nabla u|^{2}}{2}+\frac{a(x) u^{2}}{2}-G(x, u)\right), \quad \text { where } \quad G(x, t)=\int_{0}^{t} g(x, s) d s .
$$

The Nehari manifold is defined by

$$
\mathcal{N}=\left\{u \in X \backslash\{0\}: \Phi^{\prime}(u) u=0\right\} .
$$

We also define

$$
\mathcal{N}_{ \pm}=\{u \in \mathcal{N}: \pm u \geq 0\}, \quad \beta_{ \pm}=\inf \left\{\Phi(u): u \in \mathcal{N}_{ \pm}\right\}
$$

Theorem 4.1. Under the assumptions $\left(A_{1}\right)-\left(A_{6}\right)$, let $u$ be a minimizer of $\Phi$ on $\mathcal{N}_{+}$. Then $u$ is a positive (resp. negative) solution of (4.1), and there exists $i \in G_{*}$ such that $u^{*}=u \circ i$. 
Proof. Let $u \in \mathcal{N}_{+}$be such that $\Phi(u)=\beta_{+}$. Then $u$ is a solution of (4.1) (see [4] or [14]). Since for every $H \in \mathcal{H}_{*}$, one has $u^{H} \in \mathcal{N}_{+}$and $\Phi\left(u^{H}\right)=\beta_{+}$, the function $u^{H}$ is also a solution of (4.1). It then suffices to use Theorem 3.12 .

Theorem 4.2. Under assumptions $\left(A_{1}\right),\left(A_{2}\right),\left(A_{3}\right)$ with $p<2^{*},\left(A_{4}\right),\left(A_{6}\right)$, if $\Omega$ is bounded and

$$
\lim _{t \rightarrow \infty} \frac{G(x, t)}{t^{2}}=\infty
$$

uniformly in $x$, then there exists a minimizer of $\Phi$ on $\mathcal{N}_{+}$.

Proof. We omit the proof since it is simpler than the proof of Theorem 4.5 below.

\subsection{Symmetry of least energy nodal solutions}

We define

$$
\begin{aligned}
\mathcal{M} & =\left\{u \in X: u^{ \pm} \neq 0, \Phi^{\prime}\left(u^{+}\right) u^{+}=\Phi^{\prime}\left(u^{-}\right) u^{-}=0\right\}, \\
\beta & =\inf \{\Phi(u): u \in \mathcal{M}\} .
\end{aligned}
$$

Theorem 4.3. Under the assumptions $\left(A_{1}\right)-\left(A_{6}\right)$, if $u$ is a minimizer of $\Phi$ on $\mathcal{M}$, then $u$ is a nodal solution of (4.1) and there exists $i \in G_{*}$ such that $u^{*}=u \circ i$.

Remark 4.4. One can also say that $u$ has exactly two nodal domains in the following sense: $u$ cannot be decomposed as $u=u_{1}+u_{2}+u_{3}$ with $u_{i} \in \mathrm{W}_{\mathrm{loc}}^{1,1}(\Omega) \backslash\{0\}$ and $u_{i} u_{j}=0$ almost everywhere for $i \neq j$.

Proof. Let $u \in \mathcal{M}$ be such that $\Phi(u)=\beta$. Then $u$ is a solution of (4.1) with exactly two nodal domains (see [4] or [14]). Since for every $H \in \mathcal{H}_{*}, u^{H} \in \mathcal{M}$ and $\Phi\left(u^{H}\right)=\beta$, the function $u^{H}$ is also a solution of (4.1). It then suffices to use Theorem 3.12

Theorem 4.5. Under the assumptions $\left(A_{1}\right),\left(A_{2}\right),\left(A_{3}\right)$ with $p<2^{*},\left(A_{4}\right),\left(A_{6}\right)$ and $\left(A_{7}\right)$, if $\Omega$ is bounded, then there exists a minimizer of $\Phi$ on $\mathcal{M}$.

Proof. Let $\left(u_{n}\right) \subset \mathcal{M}$ be such that $\Phi\left(u_{n}\right) \rightarrow \beta$. By passing to a subsequence if necessary, we can assume that

$$
v_{n}^{+}=\frac{u_{n}^{+}}{\left\|u_{n}^{+}\right\|} \rightarrow v^{+}, \quad v_{n}^{-}=\frac{u_{n}^{-}}{\left\|u_{n}^{-}\right\|} \rightarrow v^{-},
$$

in $X$. By assumption we have

$$
\Phi\left(u_{n}^{+}\right)=\max _{t>0} \Phi\left(t u_{n}^{+}\right), \quad \Phi\left(u_{n}^{-}\right)=\max _{t>0} \Phi\left(t u_{n}^{-}\right) .
$$

Hence, for every $s, t>0$,

$$
\frac{s^{2}}{2}-\int_{\Omega} G\left(x, s v_{n}^{+}(x)\right) d x+\frac{t^{2}}{2}-\int_{\Omega} G\left(x, t v_{n}^{-}(x)\right) d x \leq \Phi\left(u_{n}\right) .
$$


We obtain, for every $s, t>0$,

$$
\frac{s^{2}}{2}-\int_{\Omega} G\left(x, s v^{+}(x)\right) d x+\frac{t^{2}}{2}-\int_{\Omega} G\left(x, t v^{-}(x)\right) d x \leq \beta .
$$

Necessarily, $v_{+} \neq 0 \neq v^{-}$. We then have, for every $s, t>0$, by lower semicontinuity,

$$
\Phi\left(s v^{+}\right)+\Phi\left(t v^{-}\right) \leq \beta .
$$

By assumption, there exist $s_{0}, t_{0}>0$ such that

$$
\Phi\left(s_{0} v^{+}\right)=\max _{s>0} \Phi\left(s v^{+}\right), \quad \Phi\left(t_{0} v^{-}\right)=\max _{t>0} \Phi\left(t v^{-}\right) .
$$

Thus $\beta=\Phi\left(s_{0} v^{+}\right)+\Phi\left(t_{0} v^{-}\right)$.

\subsection{Examples}

The results of this section can be applied to the problem

$$
\begin{cases}-\Delta u+a(x) u=b(x) f(u) & \text { in } \Omega \\ u=0 & \text { on } \partial \Omega\end{cases}
$$

where $f \in C\left(\mathbb{R} ; \mathbb{R}^{+}\right)$is such that $\lim _{s \rightarrow 0} f(s) / s=0, s \mapsto f(s) / s$ is increasing and $\lim \sup _{s \rightarrow \infty} f(s) / s^{p-1}<\infty$ for some $p<2^{*}$.

Assume that $\Omega$ is a product of a ball or an annulus of $\mathbb{R}^{k}$ with another bounded domain in $\mathbb{R}^{N-k}$. The domain $\Omega$ is then totally invariant with respect to some cap symmetrization of order $k$. Assume $a \in \mathrm{L}_{\text {loc }}^{1}(\Omega)$ and $b \in \mathrm{L}^{\infty}(\Omega)$ with $b \geq 0$ are also totally invariant, and that $-\Delta+a$ satisfies $\left(A_{6}\right)$. Then the ground state and the minimal nodal solutions of 4.2) are invariant under a cap symmetrization of order $k$, i.e. they depend at most on two variables in $\mathbb{R}^{k}$ and $N-k$ variables in $\mathbb{R}^{N-k}$.

If $\Omega=\mathbb{R}^{N}$, let $*$ be the Schwarz symmetrization with respect to 0 . Assume $a \in$ $\mathrm{L}_{\text {loc }}^{1}(\Omega)$ and $\inf a(x)>0$. Let $b: \Omega \rightarrow \mathbb{R}$ be bounded with $\inf b(x)>0$. If $a^{*}=a$ and $(-b)^{*}=-b$, let

$$
\beta=\inf _{u \in \mathrm{H}^{1}\left(\mathbb{R}^{N}\right)} \max _{t>0} \Phi(t u) .
$$

Any positive solution $u$ of 4.2 such that $\Phi(u)=\beta$ is the translate of a radial function.

\subsection{Symmetry of eigenvalues}

Theorem 3.12 applies to the symmetry of minimizers under many constraints. In this section we show how to recover well-known symmetry properties of eigenvalues. This method might be transported to nonlinear settings in which the linear approaches would fail. 
Assume $\Omega$ is a bounded domain, and let $a \in \mathrm{L}^{1}(\Omega)$. If $a_{-} \in \mathrm{L}^{N / 2}(\Omega)$, then $-\Delta+a$ has a nondecreasing sequence $\left(\lambda_{k}\right)_{k \geq 1}$ of eigenvalues characterized as

$$
\lambda_{k+1}=\inf \left\{\int_{\Omega}\left(|\nabla u|^{2}+a u^{2}\right): \int_{\Omega} u^{2}=1 \text { and } \int_{\Omega} u e_{j}=0 \text { for } 1 \leq j \leq k\right\} .
$$

One has $-\Delta u+a u=\lambda_{k+1} u$ if and only if $\int_{\Omega}\left(|\nabla u|^{2}+a u^{2}\right)=\lambda_{k+1} \int_{\Omega} u^{2}$ and $\int_{\Omega} u e_{j}=0$ for $1 \leq j \leq k$.

Proposition 4.6. Let $*$ be a spherical cap symmetrization and let $\Omega \subset \mathbb{R}^{N}$ be bounded. Assume $\Omega, e_{1}, \ldots, e_{k}$ are totally invariant with respect to $*$. If

$$
\begin{cases}-\Delta u+a u=\lambda_{k+1} u & \text { in } \Omega \\ u=0 & \text { on } \partial \Omega\end{cases}
$$

then there exists $i \in G_{*}$ such that $u^{*}=u \circ i$.

Proof. One has $\int_{\Omega}\left(|\nabla u|^{2}+a u^{2}\right)=\lambda_{k+1} \int_{\Omega} u^{2}$ and $\int_{\Omega} u e_{j}=0$ for $1 \leq j \leq k$. For every $H \in \mathcal{H}_{*}$, since $a$ and $e_{j}, 1 \leq j \leq k$, are totally invariant, we have

$$
\int_{\Omega}\left(\left|\nabla\left(u^{H}\right)\right|^{2}+a\left(u^{H}\right)^{2}\right)=\lambda_{k+1} \int_{\Omega}\left(u^{H}\right)^{2} \quad \text { and } \quad \int_{\Omega} u^{H} e_{j}=0,
$$

so that

$$
-\Delta u^{H}+a u^{H}=\lambda_{k+1} u^{H} .
$$

Since $a \in \mathrm{L}_{\text {loc }}^{1}(\Omega)$, Theorem 3.12 is applicable and yields the conclusion.

The same holds for Neumann and Robin boundary conditions. When $\Omega$ is not bounded, the variational characterization of $\lambda_{k}$ still works if one assumes $\lim _{|x| \rightarrow \infty} a(x)=\infty$, and the conclusion of Proposition 4.6 also holds.

Classical separation of variables methods give in fact results that are stronger than Proposition 4.6. The total invariance of $\Omega$ under a spherical cap symmetrization implies that it is invariant under rotations of $\mathrm{SO}(K)$ for some $1 \leq K \leq N$. A basis of eigenfunctions is given by $U_{m, l}\left(|x|, x^{\prime \prime}\right) H(x /|x|)$ with associated eigenvalues $\mu_{m, l}$, where $U_{m, l}$ is a radial eigenvalue of

$$
-\Delta U_{m, l}+\left(a+\frac{m(m+K)}{|x|^{2}}\right) U_{m, l}=\mu_{m, l} U_{m, l}
$$

and $H$ is a homogeneous harmonic polynomial of degree $m \geq 0$. The variational characterization of $\mu_{m, l}$ gives $\mu_{1,1}<\mu_{m, 1}$ for every $m>1$; in particular, the first nonradial eigenfunction is invariant under cap symmetrization.

Acknowledgments. The authors thank Augusto C. Ponce for useful discussions and suggestions.

The first author is supported by a research fellow grant of the Fonds National de la Recherche Scientifique (Belgium). 


\section{References}

[1] Ancona, A.: Une propriété d'invariance des ensembles absorbants par perturbation d'un opérateur elliptique. Comm. Partial Differential Equations 4, 321-337 (1979) Zbl 0459.35027 MR 0525774

[2] Bartsch, T.: Critical point theory on partially ordered Hilbert spaces. J. Funct. Anal. 186, 117-152 (2001) Zbl pre01679364 MR 1863294

[3] Bartsch, T., Weth, T.: A note on additional properties of sign changing solutions to superlinear elliptic equations. Topol. Methods Nonlinear Anal. 22, 1-14 (2003) Zbl pre02096714 MR 2037264

[4] Bartsch, T., Weth, T., Willem, M.: Partial symmetry of least energy nodal solutions to some variational problems. J. Anal. Math. 96, 1-18 (2005) Zbl pre05010201 MR 2177179

[5] Brezis, H., Ponce, A. C.: Remarks on the strong maximum principle. Differential Integral Equations 16, 1-12 (2003) Zbl pre02004993 MR 1948870

[6] Brezis, H., Ponce, A. C.: Kato's inequality when $\Delta u$ is a measure. C. R. Math. Acad. Sci. Paris 338, 599-604 (2004) Zbl pre02093937 MR 2056467

[7] Brock, F., Solynin, A. Yu.: An approach to symmetrization via polarization. Trans. Amer. Math. Soc. 352, 1759-1796 (2000) Zbl 0965.49001 MR 1695019

[8] Castro, A., Drábek, P., Neuberger, J. M.: A sign-changing solution for a superlinear Dirichlet problem. II. In: Proc. Fifth Mississippi State Conf. on Differential Equations and Computational Simulations, Electron. J. Differ. Equ. Conf. 10, Southwest Texas State Univ., 101-107 (2003) Zbl pre01911317 MR 1976635

[9] Chabi, A., Haraux, A.: Un théorème de valeurs intermédiaires dans les espaces de Sobolev et applications. Ann. Fac. Sci. Toulouse Math. (5) 7, 87-100 (1985) Zbl 0557.35055 MR 0842764

[10] Chern, J.-L., Lin, C.-S.: The symmetry of least-energy solutions for semilinear elliptic equations. J. Differential Equations 187, 240-268 (2003) Zbl pre01884221 1 MR 1949440

[11] Evans, L. C., Gariepy, R. F.: Measure Theory and Fine Properties of Functions. Studies in Advanced Mathematics, CRC Press, Boca Raton, FL (1992) Zbl 0804.28001 MR 1158660

[12] Lieb, E. H., Loss, M.: Analysis. 2nd ed., Grad. Stud. Math. 14, Amer. Math. Soc., Providence, RI (2001) Zbl 0966.26002 MR 1817225

[13] Lin, C.-S., Takagi, I.: Method of rotating planes applied to a singularly perturbed Neumann problem. Calc. Var. Partial Differential Equations 13, 519-536 (2001) Zbl pre01713845 MR 1867940

[14] Liu, Z., Wang, Z.-Q.: On the Ambrosetti-Rabinowitz superlinear condition. Adv. Nonlinear Stud. 4, 563-574 (2004) Zbl pre02149279 MR 2100913

[15] Smets, D., Willem, M.: Partial symmetry and asymptotic behavior for some elliptic problems. Calc. Var. Partial Differential Equations 18, 57-75 (2003) Zbl pre01993491| MR 2001882

[16] Van Schaftingen, J.: Approximation of symmetrizations and symmetry of critical points. Topol. Methods Nonlinear Anal. 28, 61-85 (2006) Zbl 1110.28014 MR 2262256

[17] Van Schaftingen, J.: Universal approximation of symmetrizations by polarizations. Proc. Amer. Math. Soc. 134, 177-186 (2006) Zbl pre02212306 MR 2170557

[18] Van Schaftingen, J., Willem, M.: Set transformations, symmetrizations and isoperimetric inequalities. In: Nonlinear Analysis and Applications to the Physical Sciences, V. Benci and A. Masiello (eds.), Springer, 135-152 (2004) MR 2085832

[19] Willem, M.: Analyse fonctionnelle élémentaire. Cassini, Paris (2003) Zbl pre02243106 MR 1374790 